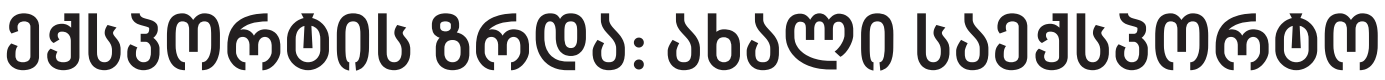

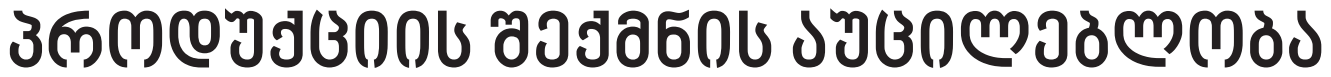

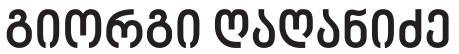

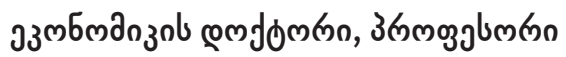

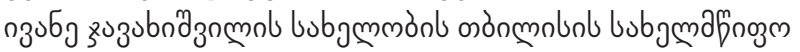

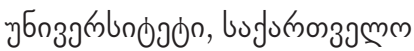

giorgi.gaganidze@tsu.ge

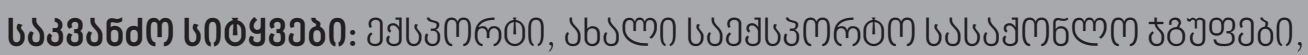

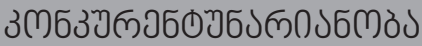

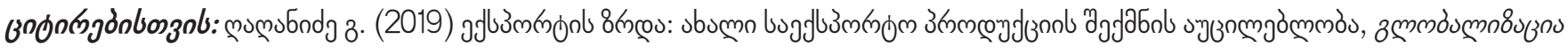
sou do86jlon, №7, 33. 24-31. https://doi.org/10.35945/gb.2019.07.003

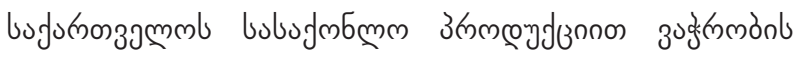

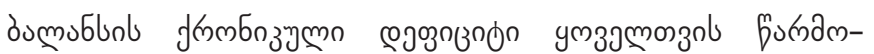

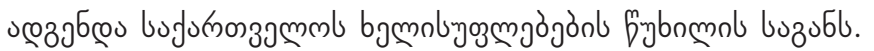

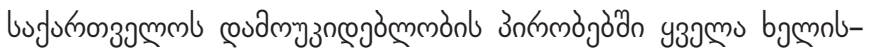

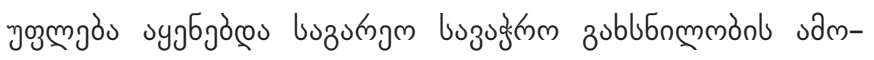

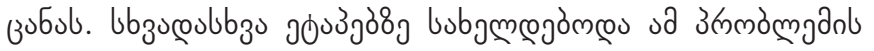

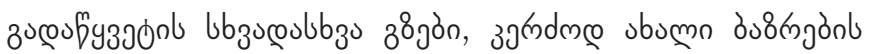

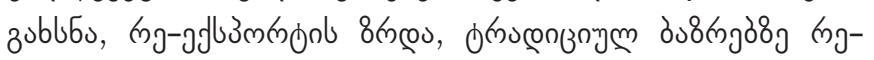

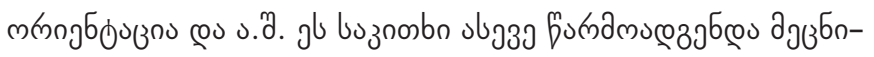

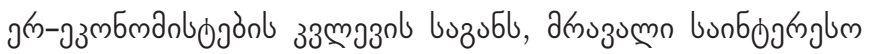

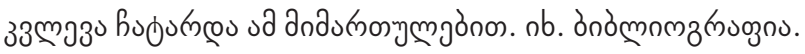

uмб yзэмs

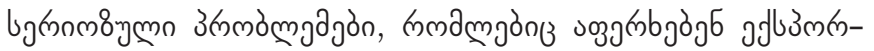

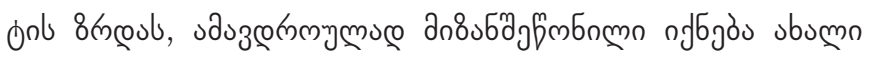

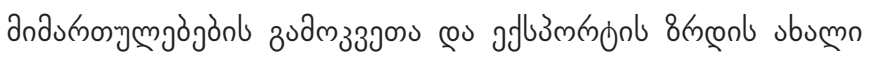

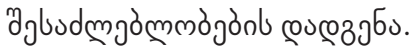

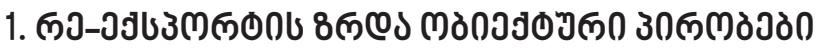

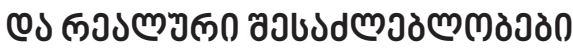

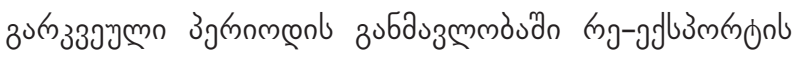

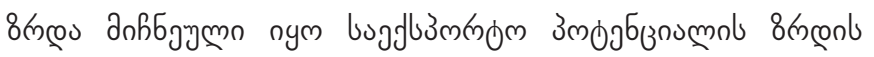

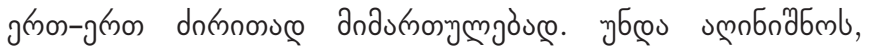

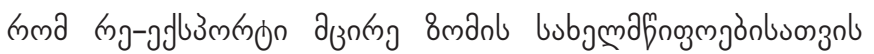

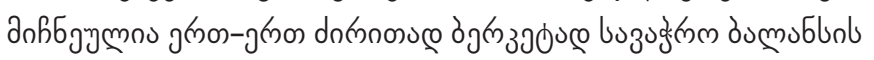

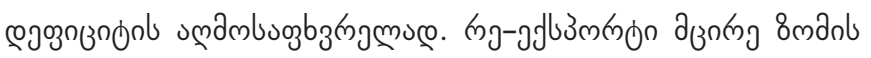

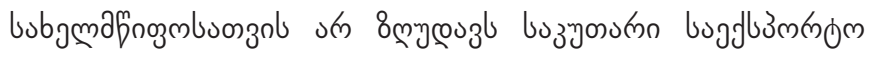

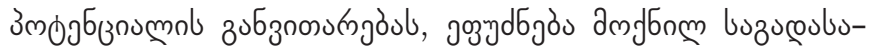

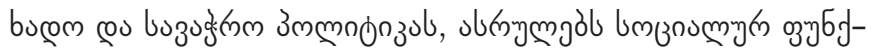

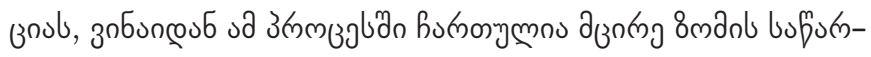

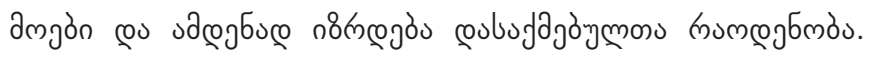

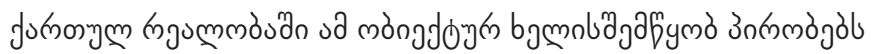

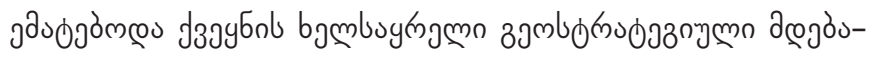

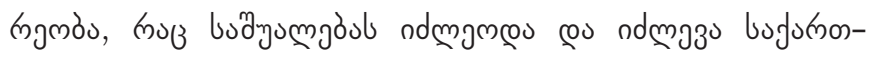

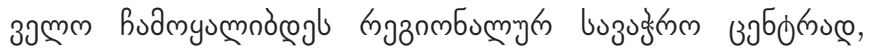

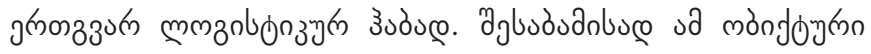

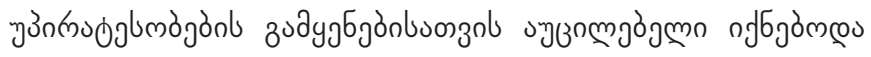

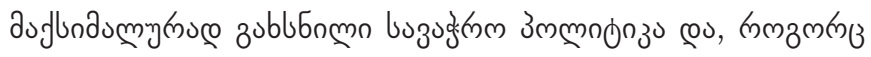

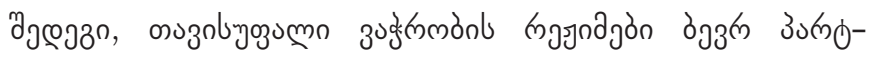

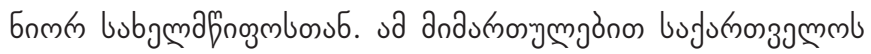

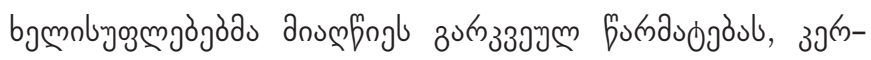

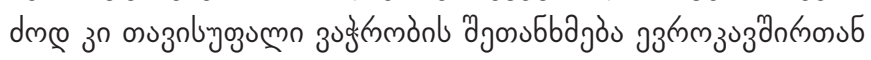

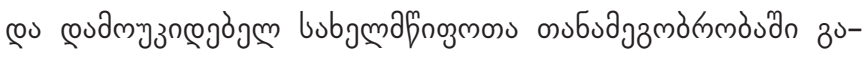

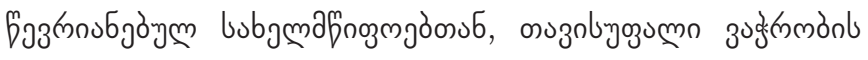

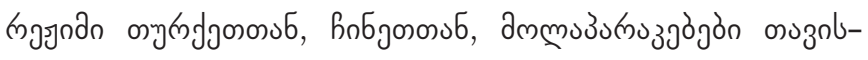

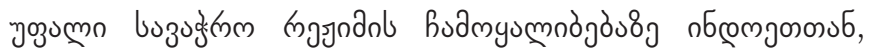

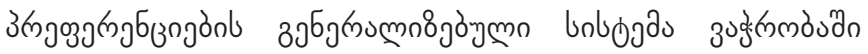

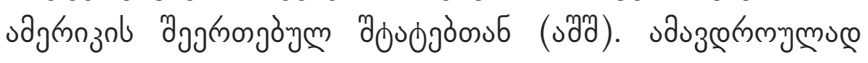

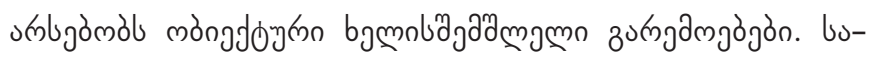

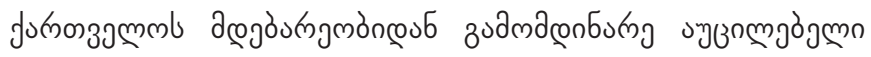

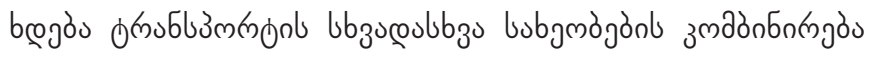

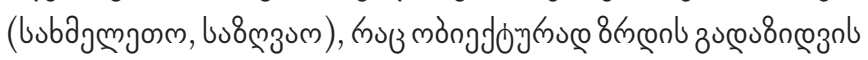

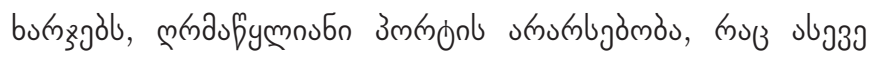




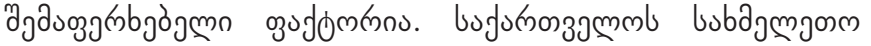

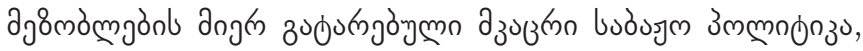

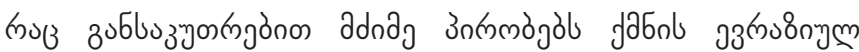

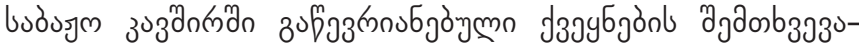

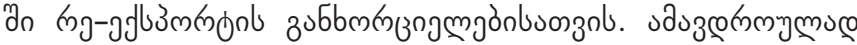

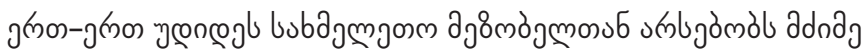

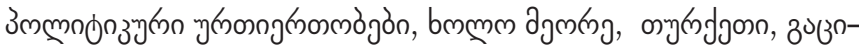

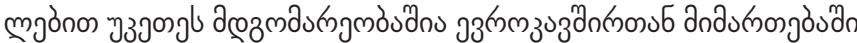

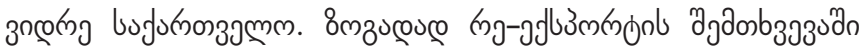

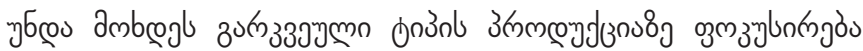

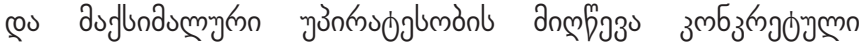

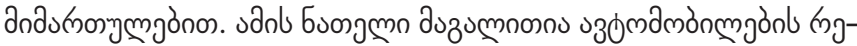

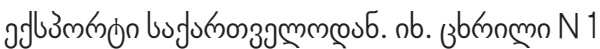

उb)

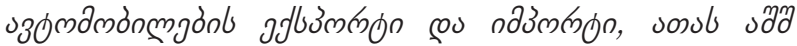
compungidon

\begin{tabular}{|c|c|c|c|c|c|c|c|c|c|c|}
\hline fagmo & 2009 & 2010 & 2011 & 2012 & 2013 & 2014 & 2015 & 2016 & 2017 & 2018 \\
\hline 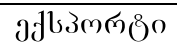 & 78,462 & 227,360 & 450,297 & 587,296 & 703,817 & 517,787 & 179,646 & 166,634 & 234,885 & 278 \\
\hline одзмп & 304,364 & 401,980 & 510,679 & 662,599 & 710,493 & 715,051 & 468,136 & 475,899 & 474,360 & 1,596 \\
\hline
\end{tabular}

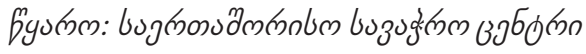

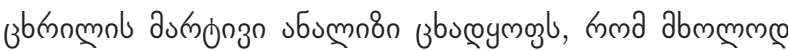

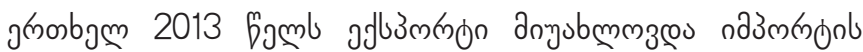

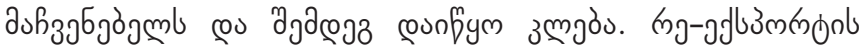

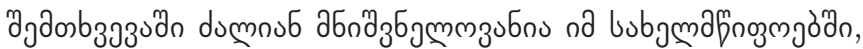

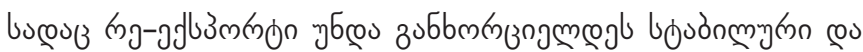

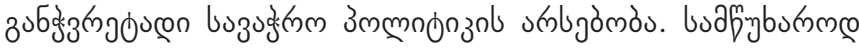

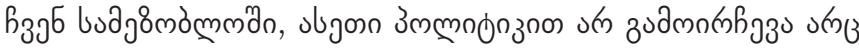
grnon bubjgmafpogn.

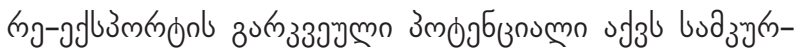

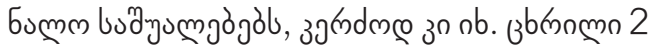

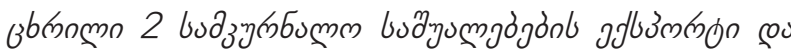

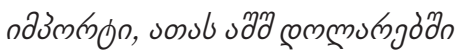

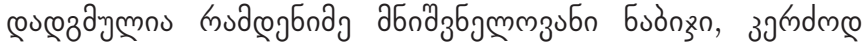

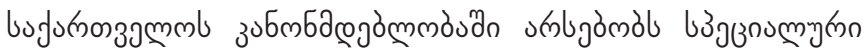

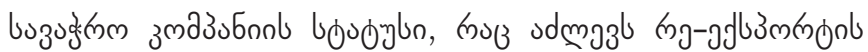

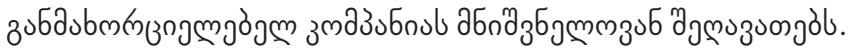

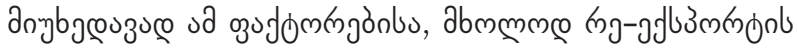

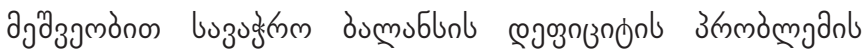

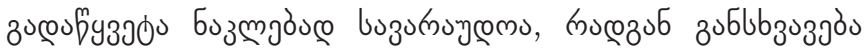

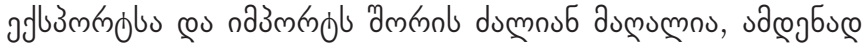

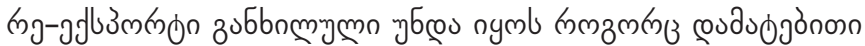
bymäa

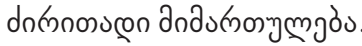

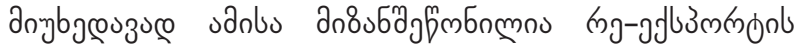

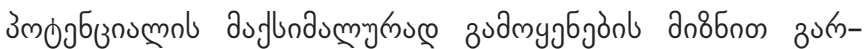

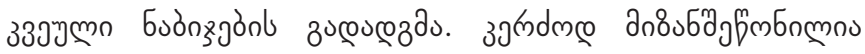

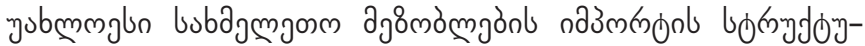

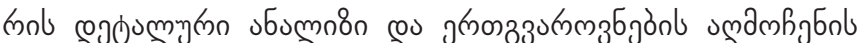

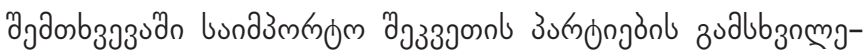

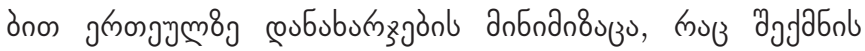

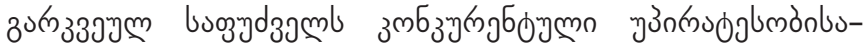

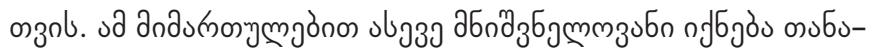

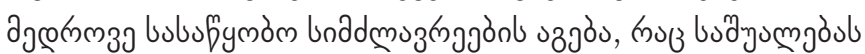

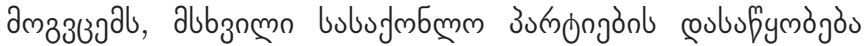

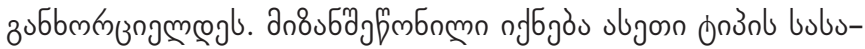

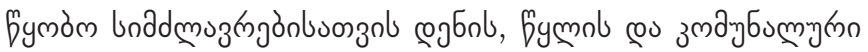

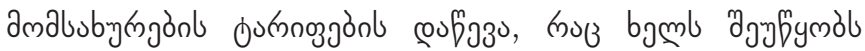

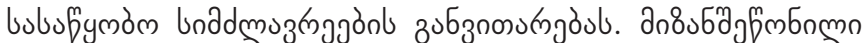

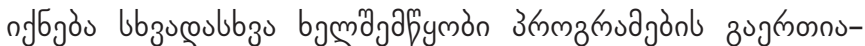

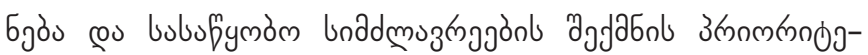

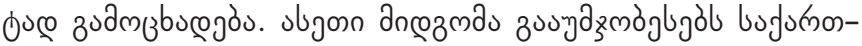

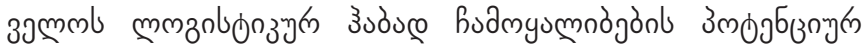
நjbudmgàmmòjal.

\begin{tabular}{|c|c|c|c|c|c|c|c|c|c|c|}
\hline Famo & 2009 & 2010 & 2011 & 2012 & 2013 & 2014 & 2015 & 2016 & 2017 & 2018 \\
\hline adb3 & 5,38 & 720 & 44,1 & 55,249 & 59,000 & 104,703 & 154,389 & 118,798 & 152,437 & 112 \\
\hline 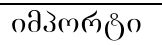 & 199,425 & 21,043 & 28,481 & 264,556 & 318,269 & 364,797 & 790,728 & 314,165 & 392,42 & 285,028 \\
\hline
\end{tabular}

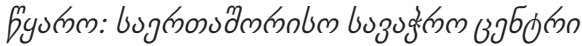

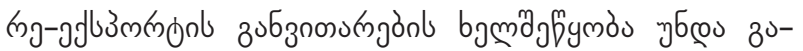

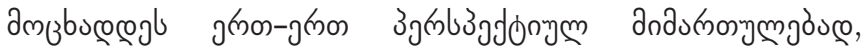

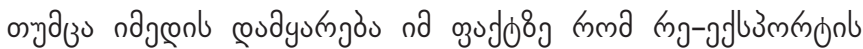

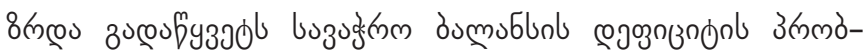

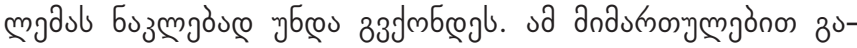

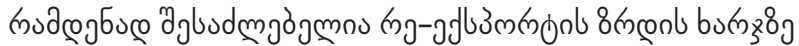

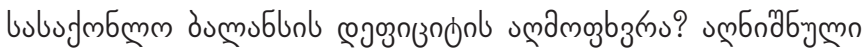

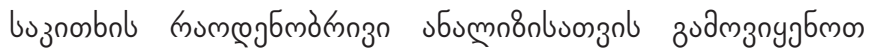

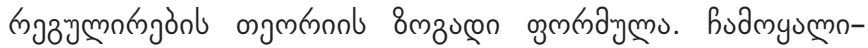

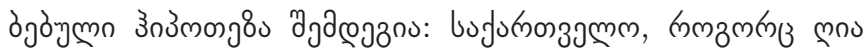

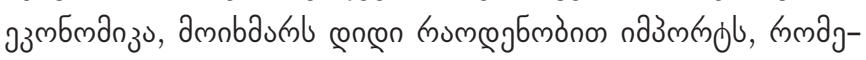

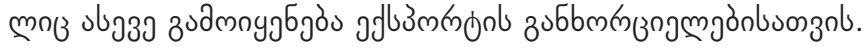




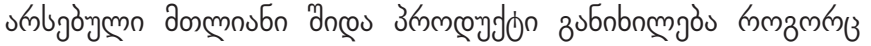

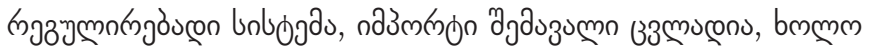

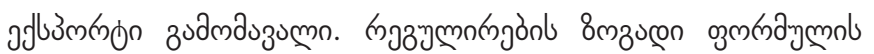

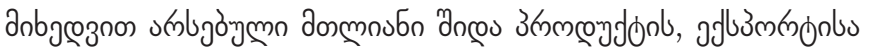

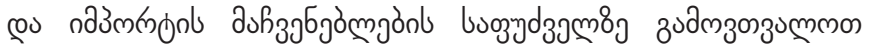

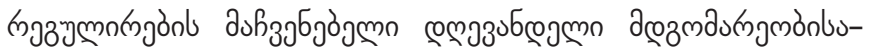

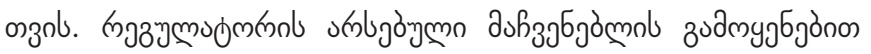

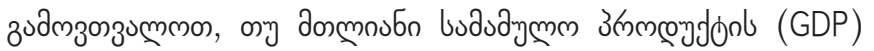

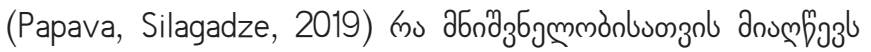

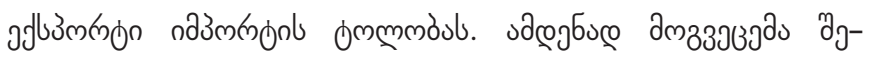

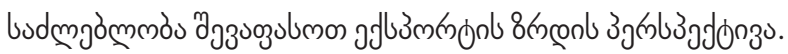

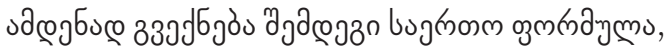 \\ $\mathrm{Y}=\mathrm{S} /(1-\mathrm{SXR}) \mathrm{X}$, \\ $Y$ jلjb3mtron \\ $X$ oдзminon

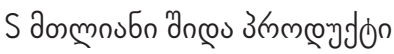

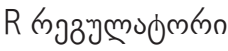

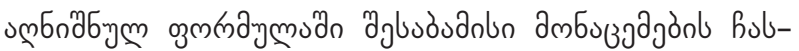

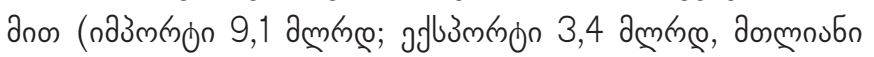

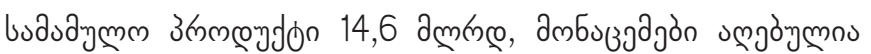

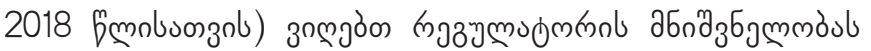

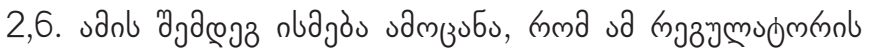

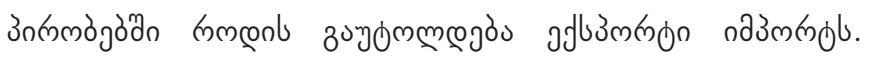

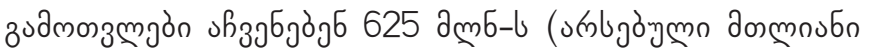

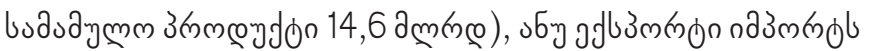

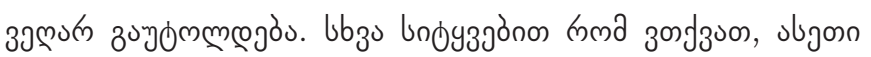

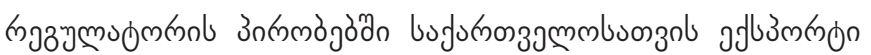

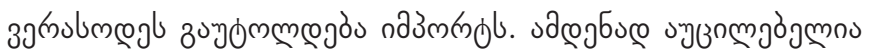

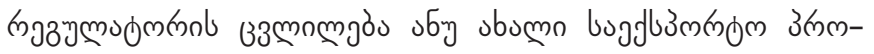

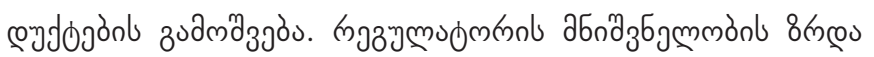

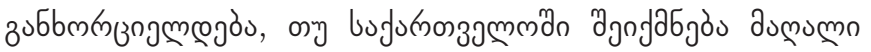

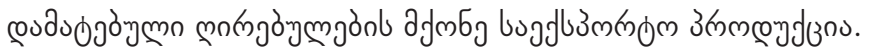

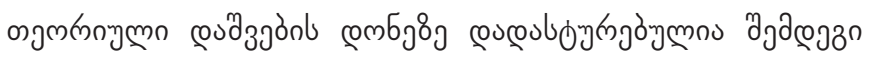

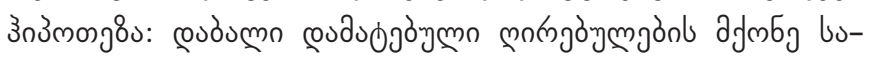

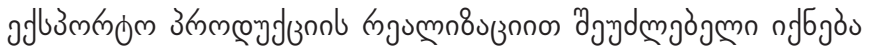

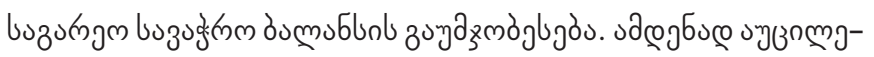

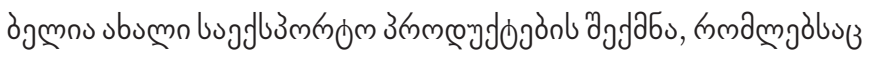

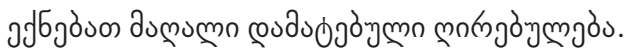

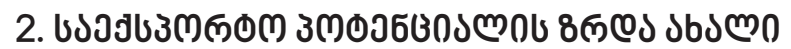

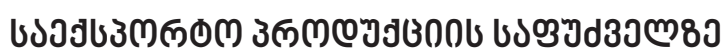

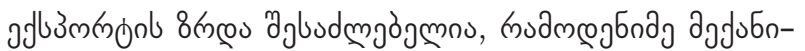
8anl aj

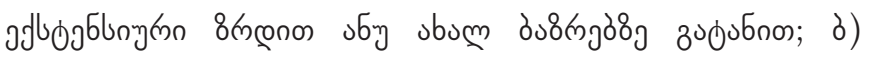

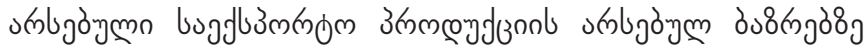

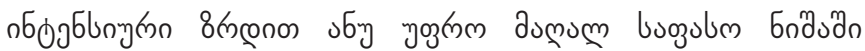

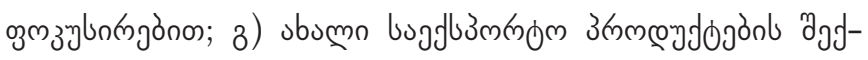

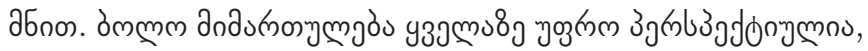

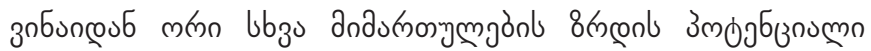

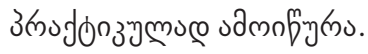

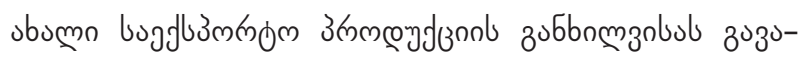

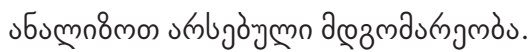

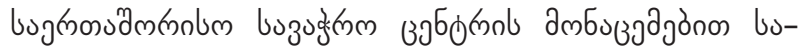

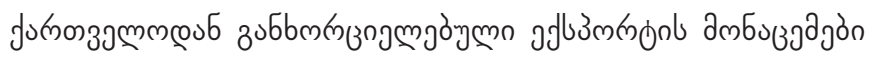

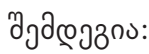

\begin{tabular}{|c|c|c|c|}
\hline & 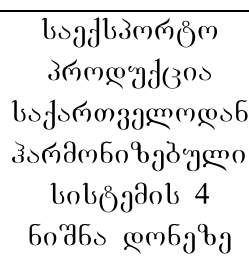 & 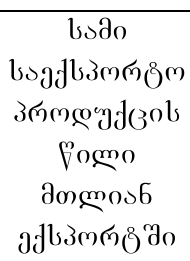 & 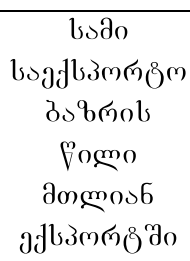 \\
\hline $\begin{array}{l}2016 \\
\text { fagmo }\end{array}$ & 1735 & 30,9 & 26,0 \\
\hline
\end{tabular}

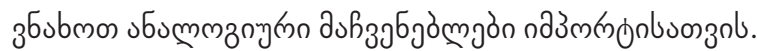

\begin{tabular}{|c|c|c|c|}
\hline & 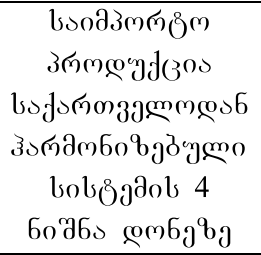 & 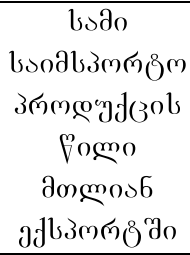 & 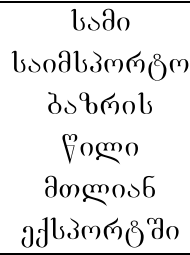 \\
\hline 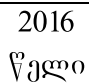 & 3801 & 12,9 & 35,6 \\
\hline
\end{tabular}

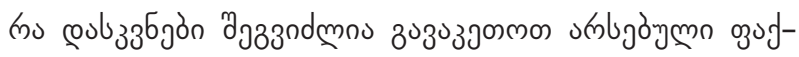

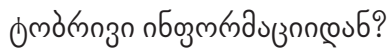

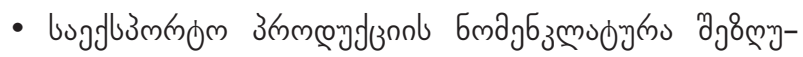
eymas;

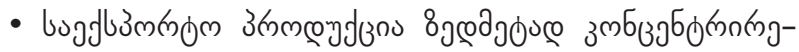

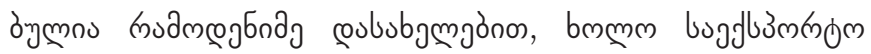

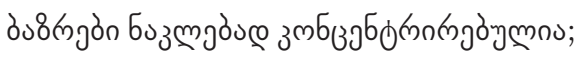

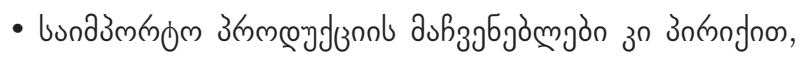

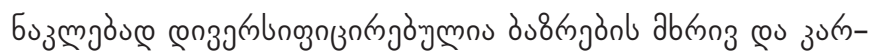

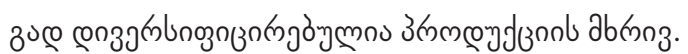

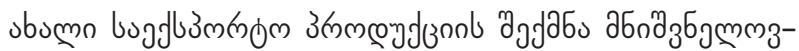

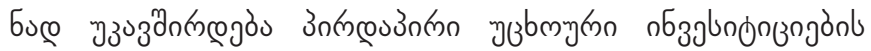

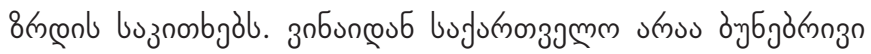

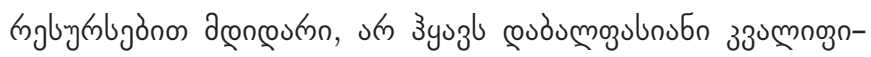

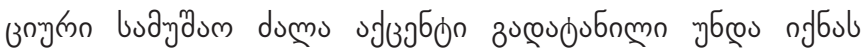

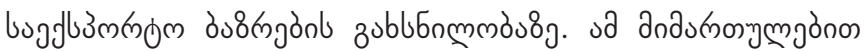

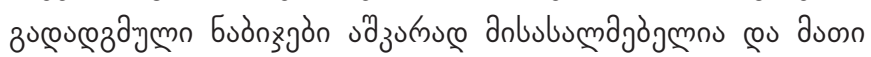

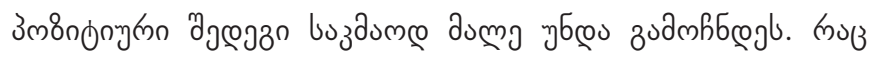




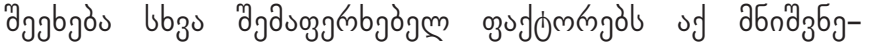

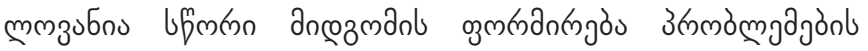

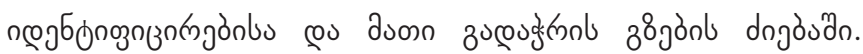

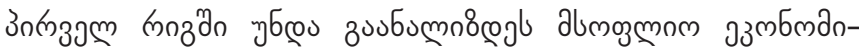

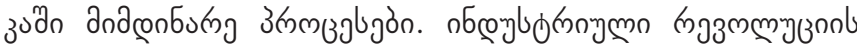

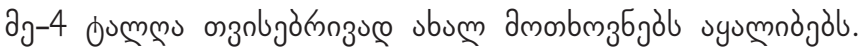

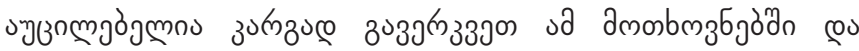

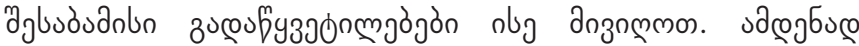

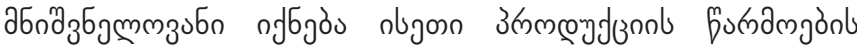

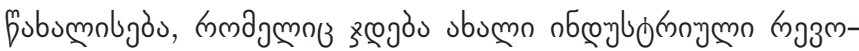
minzonb humbngiòn.

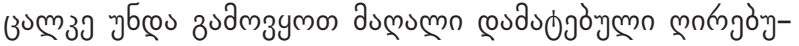

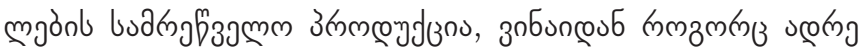

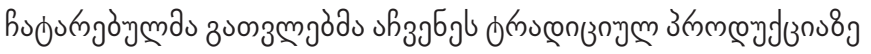

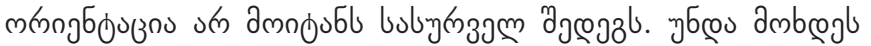

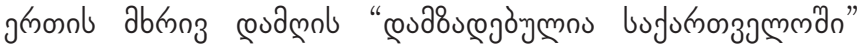

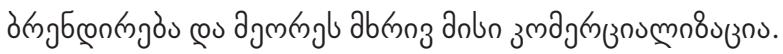

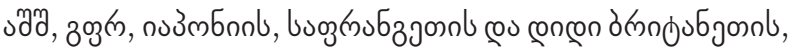

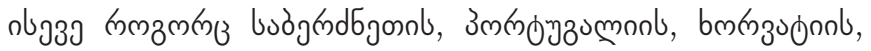

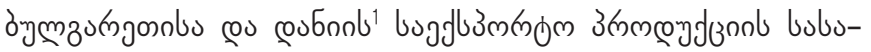

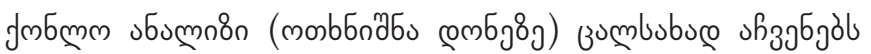

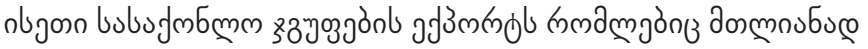

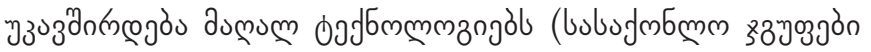

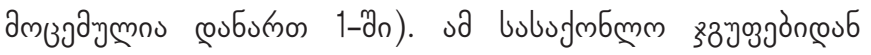

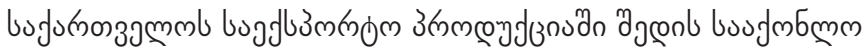

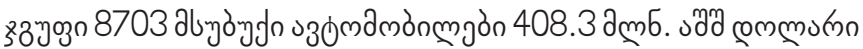

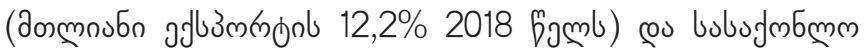

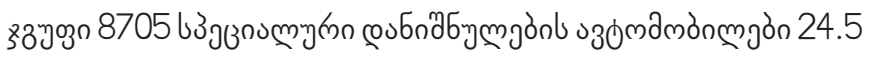

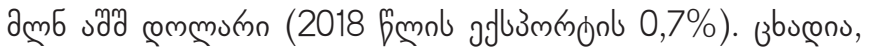

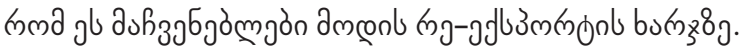

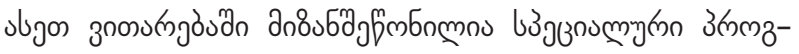

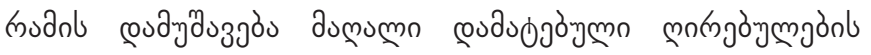

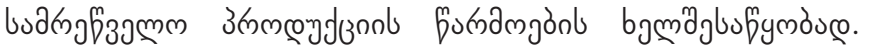

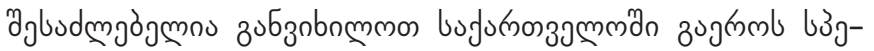

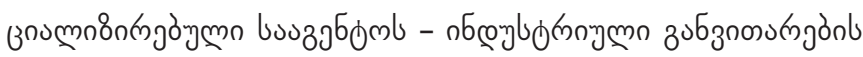

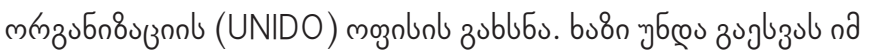

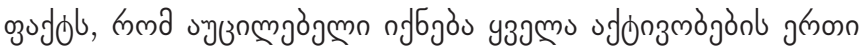

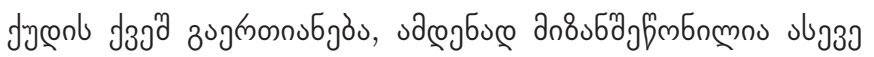

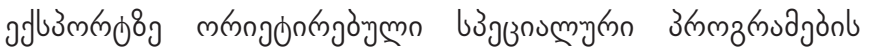

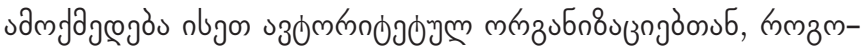

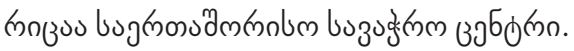

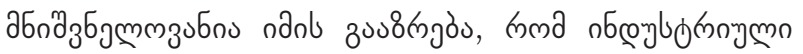

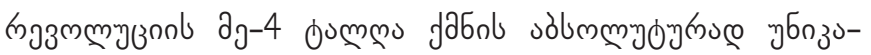

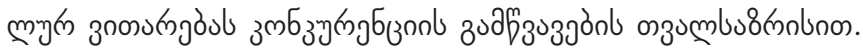

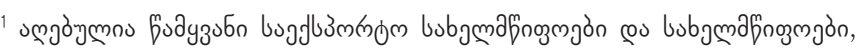

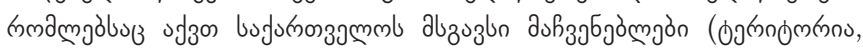

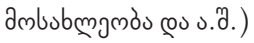

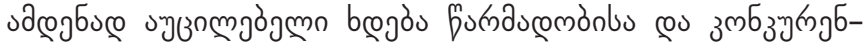

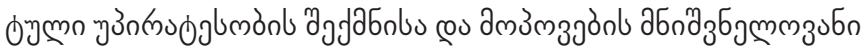

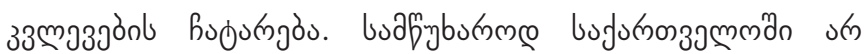

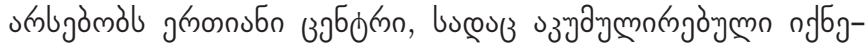

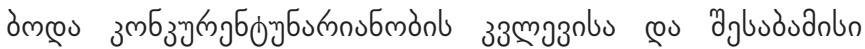

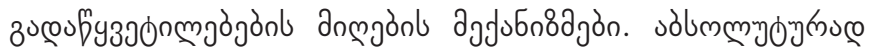

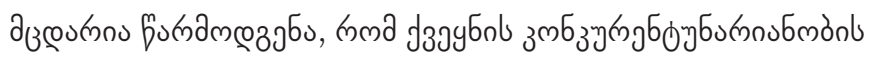

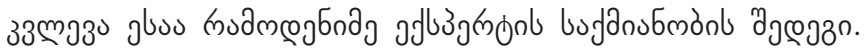

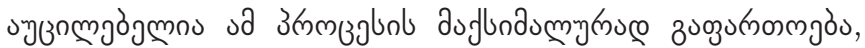

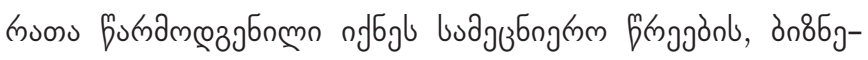

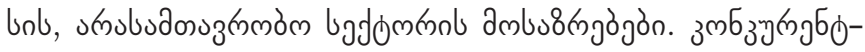

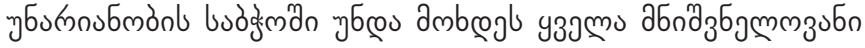

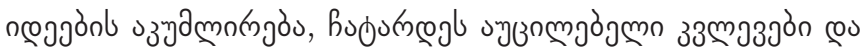

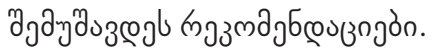

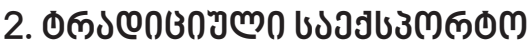

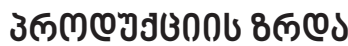

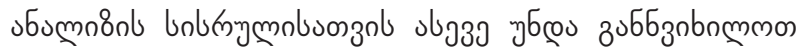

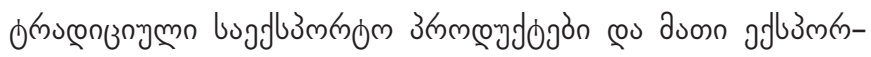

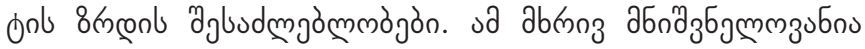

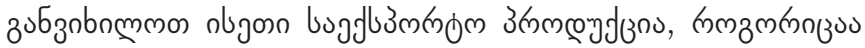

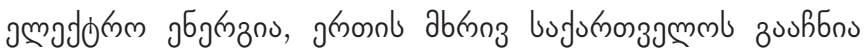

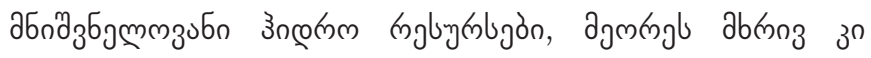

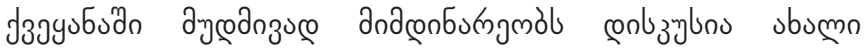

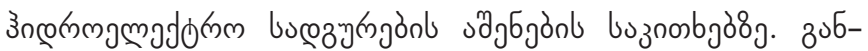

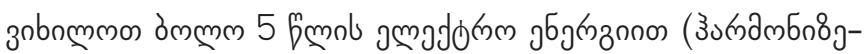

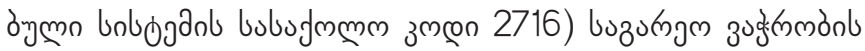
oumublon. zbrnnmo 3.

\section{6hongm 3}

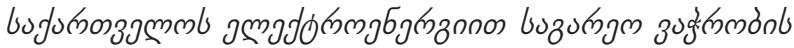

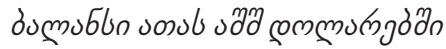

\begin{tabular}{|c|c|}
\hline 6ymo & sımsblon \\
\hline 2014 & $-21,562$ \\
\hline 2015 & $-17,756$ \\
\hline 2016 & 683 \\
\hline 2017 & $-44,518$ \\
\hline 2018 & $-56,756$ \\
\hline
\end{tabular}

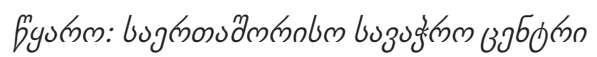

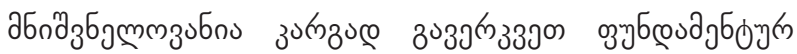

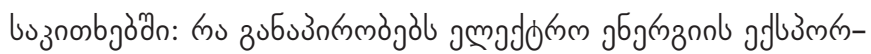

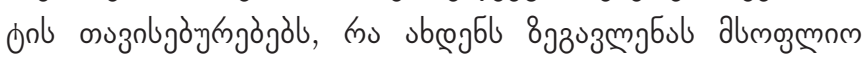

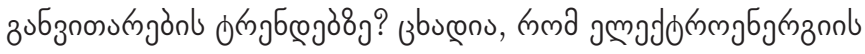




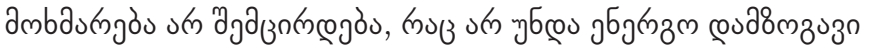

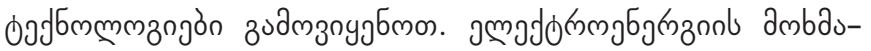

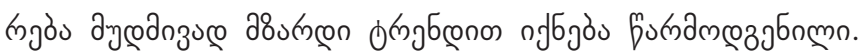

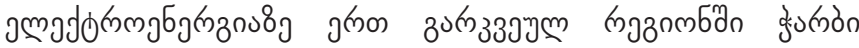

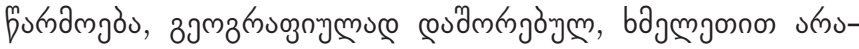

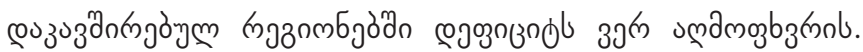

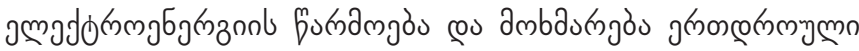

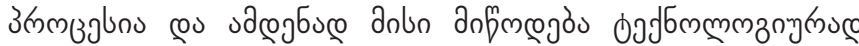

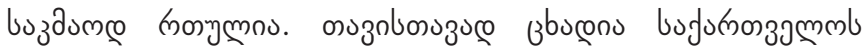

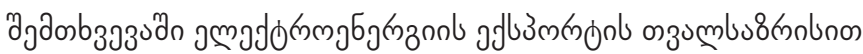

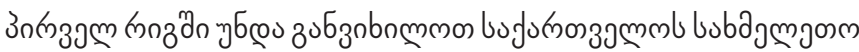

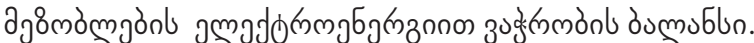

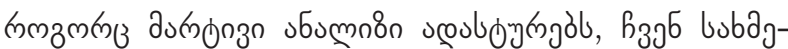

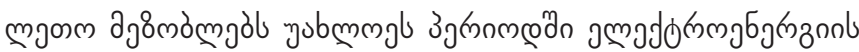

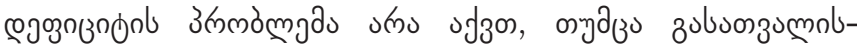

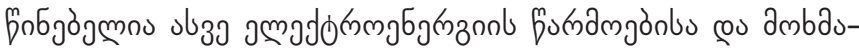

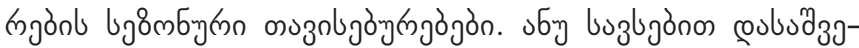

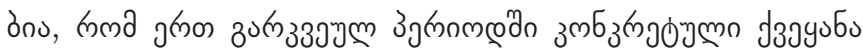

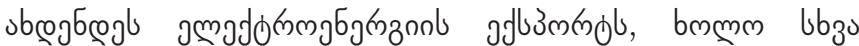

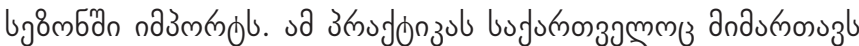

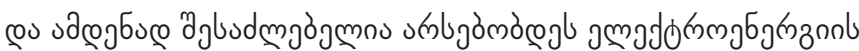

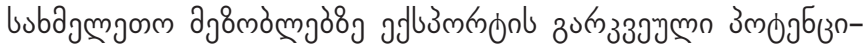

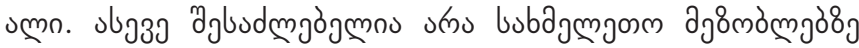

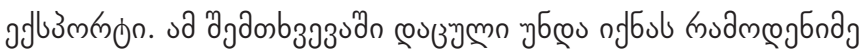

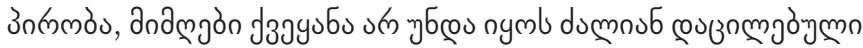

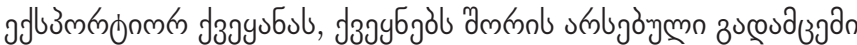

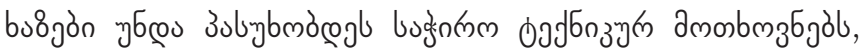

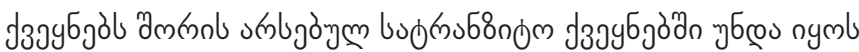

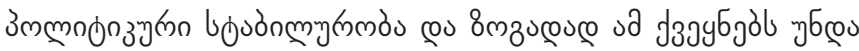

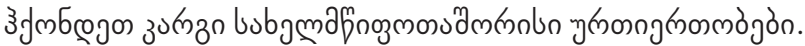

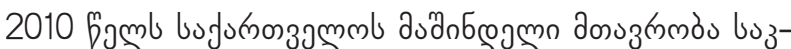

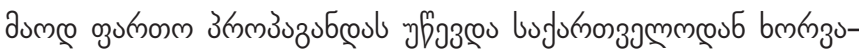
ono⿰氵 jmadd

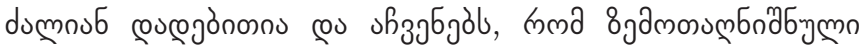

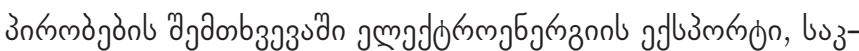

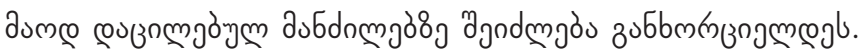

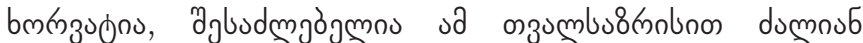

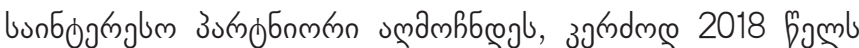

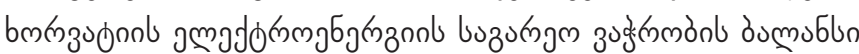

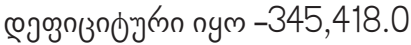

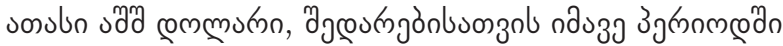

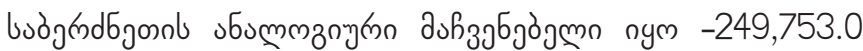
sosubn uag commutron.

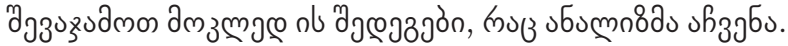

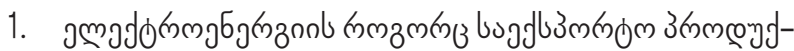

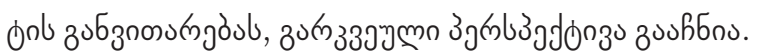

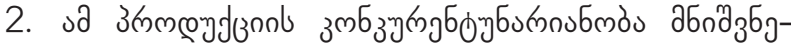

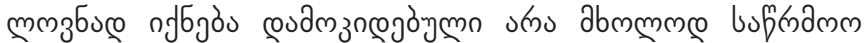

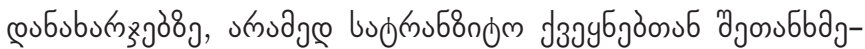
og

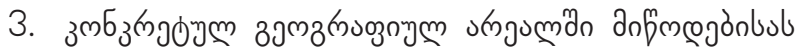

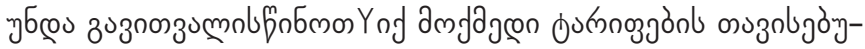

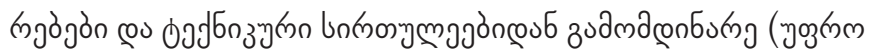

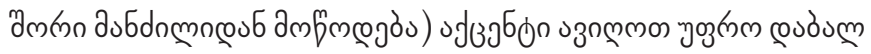

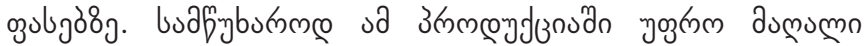
bumnlubnon zmb

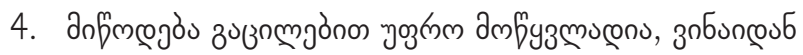

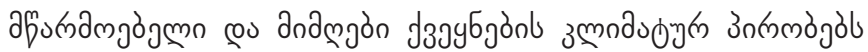

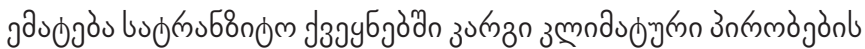

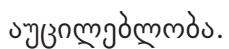

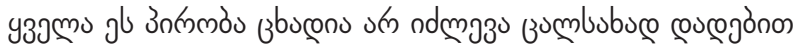

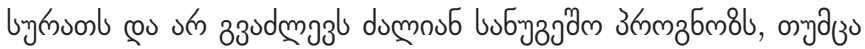

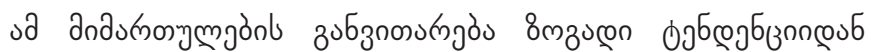

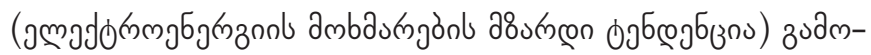

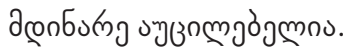

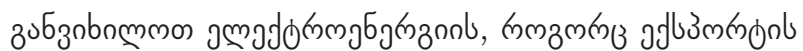

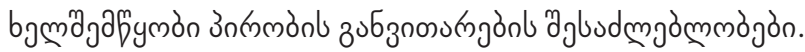

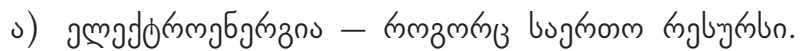

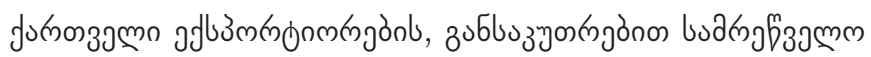

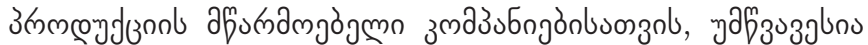

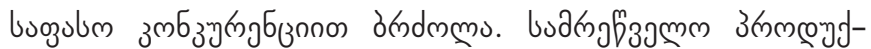

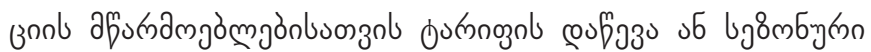

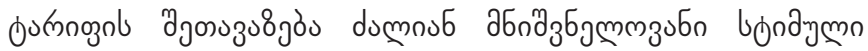

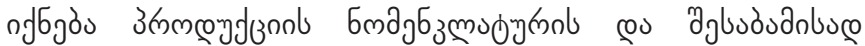

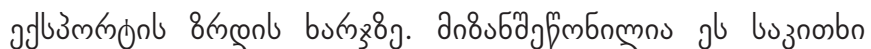

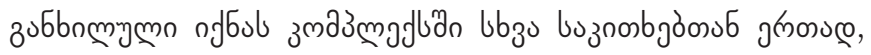

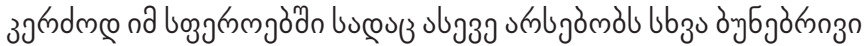

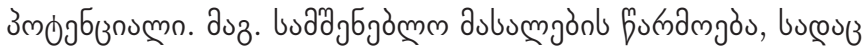

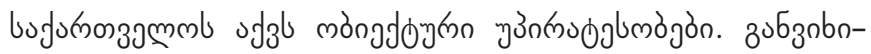

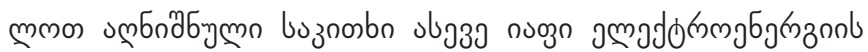

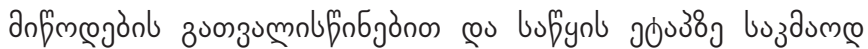

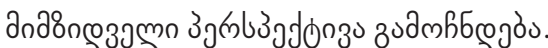

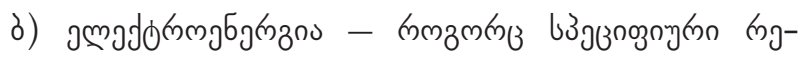

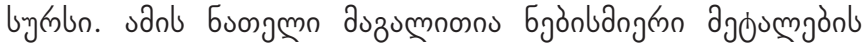

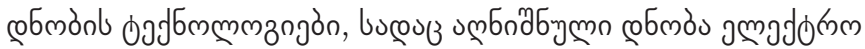

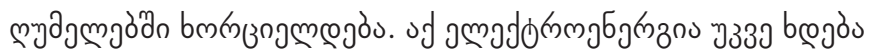

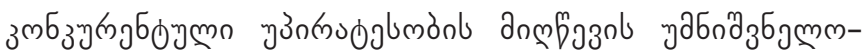

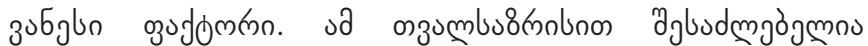

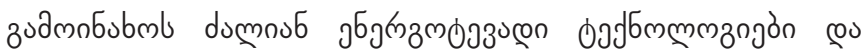

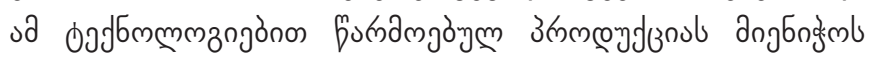

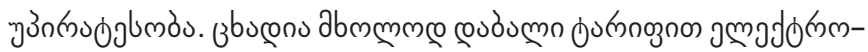




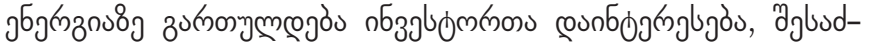

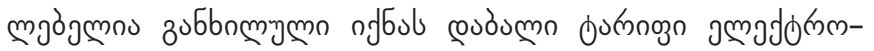

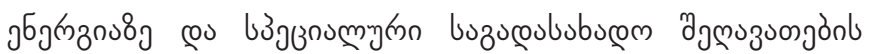

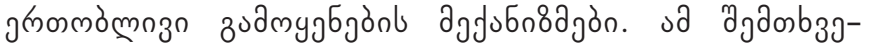

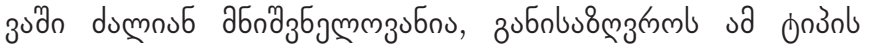

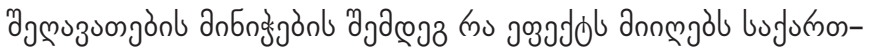

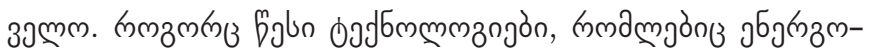

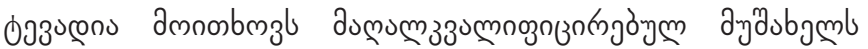

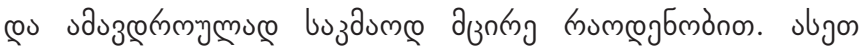

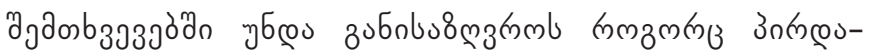

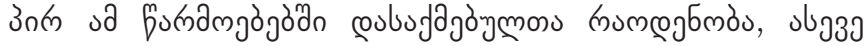

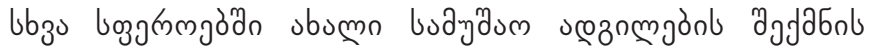

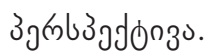

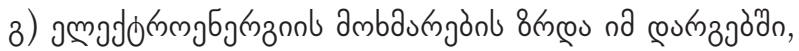

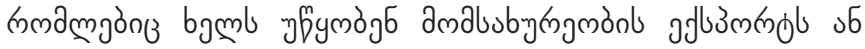

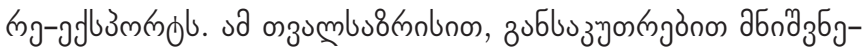

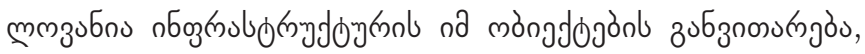

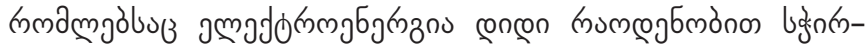

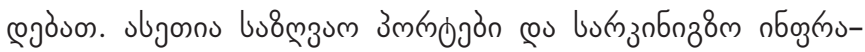

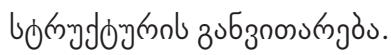

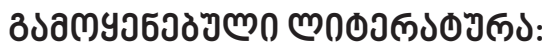

1. O'Cass, Aron and Craig Juliann (2003), "Examining Firm and Environmental Influences on Export Marketing Mix Strategy and Export Performance of Australian Exporters". European Journal of Marketing, 37 9314) 366-84

2. Papava, Vladimer, Retroeconomics - Moving from Dying to Brisk Economy, Journal of Reviews on Global economics, 2017, 6, pp 455-462

3. Papava, V. (2013). Economic Reforms in Post-Communist Georgia: Twenty Years After. New York: Nova Science Publishers.

4. Papava V., Silagadze A., 2019. "How the Term the "Gross Domestic Product" should be translated into Georgian." Globalization and Business, No. 7, pp. 203-204. (In Georgian.)

5. Gaganidze, G. (2016). Georgian Export Potential Utilization on the EU Market, Journal of International Management Studies, Volume16, Number 1.

6. Gaganidze, G. (2015). Export Potential and Competitive Advantage, scientific and practical journal, Economics and Business, N3.

7. Gaganidze,G. (2015). Export Potential of Georgian Agricultural Products on the EU Market (Based on Competitive Advantages and Market Entry Modes), European Journal of Business Research, Volume 15, Number 2, ISSN326:1945-2977.

8. Gaganidze,G.(2014) Competitive advantages of Georgian non-agricultural products on the EU Market, International Academic Conference, Business and Globalization, Dubai, 2-3rd February, ABRM, ISBN 2047-2854

9. Gaganidze,G.(2014). Competitive Advantage of Georgian Agricultural Products on the EU Market, International Academic Conference on Economics, Management and Marketing in Prague, (IAC-EMM 2014) 7-8, August, Conference proceedings, ISBN 978-80-905442-6-0

10. Gaganidze,G. (2014). Systematic Approach to Research the Competitive Advantage, TSU, scientific and practical journal, Economics and Business, N4, 2014

11. Gaganidze,G. (2014). Determining the production export potential index, TSU, scientific and practical journal, Economics and Business, N1, January-February.

12. Gaganidze,G.(2013). Competitiveness of Georgian agro products and creation of export strategies; II International Scientific-Practical Conference, Bioeconomy and Sustainable Development of Agriculture, October, Tbilisi, Proceedings 13. Gaganidze,G.(2013). New Challenges in Managing International Companies, Global Business Conference Proceedings, ISSN 1848-2252;p.73.(on English)

14. Gaganidze,G. (2013).Competitiveness of Georgian Agro products and creation of the export strategies on the EU market, TSU, scientific and practical journal, Economics and Business, N6November-December.

15.Silagadze, A., Zubiashvili, T. (2015). Parameters of the European Union and the Post-Soviet Georgia's Economy. Refereed International Journal of Business and Management Studies (IJBMS), pp. 441-448.

16.Silagadze, A., Atanelishvili,T. (2014). The main economic indicators of the EU and Georgia "Topical problems of the development of economy and economic science." Collection of scholarly works of Paata Gugushvili Institute of Economics TSU, pp.50-52. 


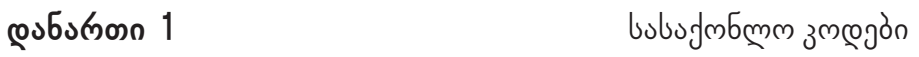

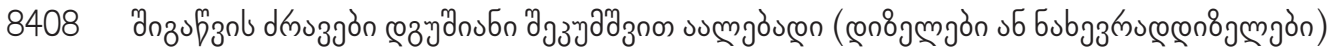

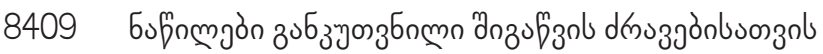

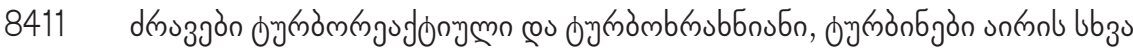

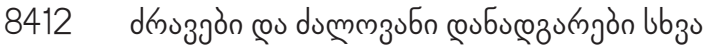

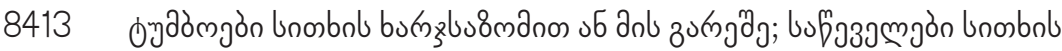

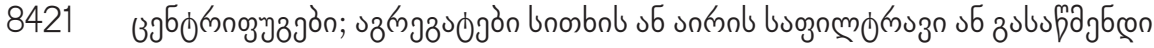

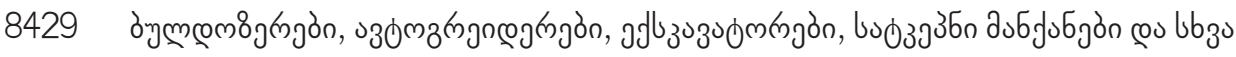

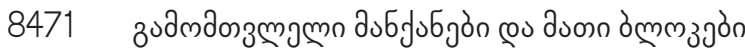

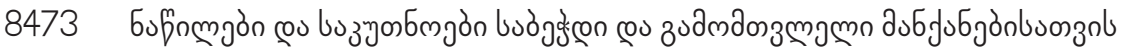

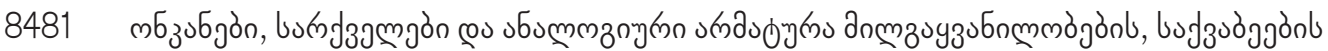

8535 د3دmod

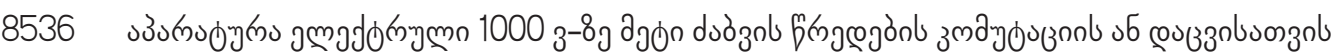

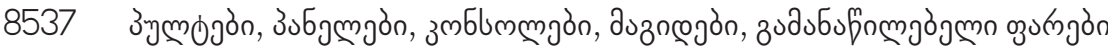

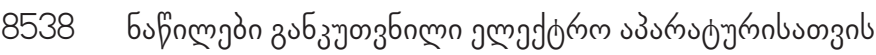

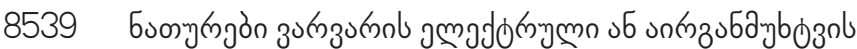

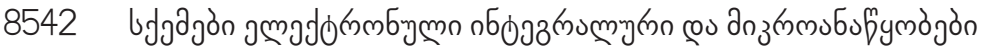

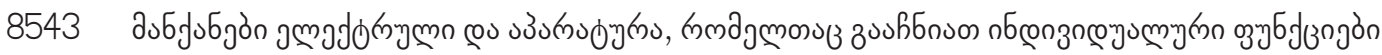

8544 aszongmgòn n8mmnn

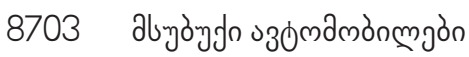

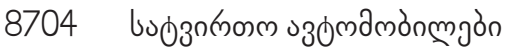

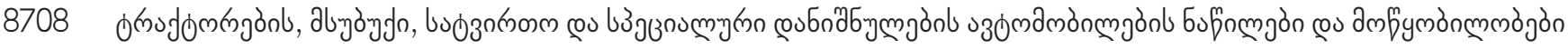

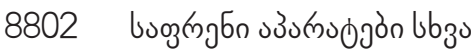

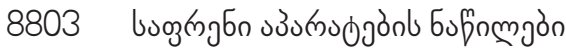




\section{GROWTH OF EXPORT: THE NEED TO CREATE NEW EXPORT PRODUCTS}

\section{GIORGI GAGANIDZE}

\section{Doctor of Economics, Professor}

Ivane Javakhishvili Tbilisi State University, Georgia

giorgi.gaganidze@tsu.ge

\section{KEYWORDS: EXPORT; NEW EXPORT COMMODITY GROUPS; COMPETITIVENESS.}

For citation: Gaganidze G. (2019), Growth Of Export: The Need To Create New Export Products, Globalization And Business, №7, pp. 24-31. (In Georgian). https://doi.org/10.35945/gb.2019.07.003

\section{SUMMARY}

In this article, the author tries to prove the advantages of exporting the export price. It is proposed to evaluate export growth based on the common formula of regulation.

The chronic shortage of balance of trade in commodity products of Georgia was always the subject of concerns of Georgian authorities. Under the independence of Georgia all governments have set a goal of foreign trade openness. At different stages, different ways of solving this problem, name- ly opening new markets, increasing re-export, re-orientation in traditional markets, etc. This issue was also a subject of research for scientists and researchers, and many interesting researches were conducted in this direction. See Bibliography.

All these directions are distinguished in this study and are considered to be the most serious problems that impede export growth, at the same time it is expedient to identify new directions and establish new opportunities for export growth. 